\title{
Hormone replacement therapy in BRCA mutation carriers and risk of ovarian, endometrial, and breast cancer: a systematic review
}

\author{
D. Huber ${ }^{1} \cdot$ S. Seitz ${ }^{1} \cdot$ K. Kast ${ }^{2} \cdot$ G. Emons ${ }^{3} \cdot$ O. Ortmann ${ }^{1}$ (I)
}

Received: 16 December 2020 / Accepted: 1 April 2021 / Published online: 22 April 2021

(c) The Author(s) 2021

\begin{abstract}
Purpose $B R C A$ mutation carriers have an increased risk of developing breast or ovarian cancer. Risk-reducing bilateral salpingo-oophorectomy (RRBSO) is associated with a decrease in risk for tubal and ovarian cancer. Hormone replacement therapy (HRT) may increase breast, ovarian, and endometrial cancer risk in the general population. This review analyses the published data on HRT and risk of cancer in BRCA mutation carriers with and without RRBSO.

Methods We included all relevant articles published in English from 1995 to October 2020. Sources were identified through a search on PubMed and Cochrane Library.

Results We included one case-control and one retrospective cohort study on ovarian and one case-control study on endometrial cancer risk and HRT in $B R C A$ mutation carriers. Regarding breast cancer risk, one case-control study on $B R C A$ mutation carriers with and without RRBSO and one case-control study, one Markov chain decision model, two prospective cohort studies, and one metaanalysis on carriers after RRBSO were included. For ovarian cancer, results were ambiguous. For breast cancer, most studies did not find an adverse effect associated with HRT. However, some of the studies found a risk modification associated with different formulations and duration of use.

Conclusion Although data are limited, HRT does not seem to have a relevant effect on cancer risk in BRCA mutation carriers. RRBSO should not be postponed to avoid subsequent HRT in this population. Adequate HRT after RRBSO should be offered to avoid chronic diseases resulting from low estrogen levels. However, further data on the safety of different formulations are needed.
\end{abstract}

Keywords Hormone replacement therapy $\cdot$ Breast cancer $\cdot$ Ovarian cancer $\cdot B R C A 1 \cdot B R C A 2$

\section{Introduction}

Hormone replacement therapy (HRT) is the most effective treatment for climacteric symptoms. Furthermore, adequate HRT may avoid chronic diseases resulting from low estrogen levels such as osteoporosis and myocardial infarction,

D. Huber and S. Seitz contributed equally.

O. Ortmann

olaf.ortmann@klinik.uni-regensburg.de

1 Department of Gynecology and Obstetrics, University Medical Center Regensburg, Regensburg, Germany

2 Center for Hereditary Breast and Ovarian Cancer, University Hospital of Cologne, Cologne, Germany

3 Department of Gynecology and Obstetrics, Georg August University Göttingen, University Medicine, Göttingen, Germany especially in younger women with premature ovarian failure. (S3-Leitlinie Peri 2020) However, HRT may increase breast, ovarian, and endometrial cancer risk in the general population. The extent of the increase in risk depends on type and duration of HRT.

A recent metaanalysis shows a significant time dependent increase in breast cancer risk associated with HRT. The increase in risk was higher for combined estrogen-progestin therapy (EPT) than for estrogen-only therapy (ET). (Collaborative Group on Hormonal Factors in Breast Cancer 2019) In hysterectomized women, ET is, therefore, recommended, when HRT is needed. Recent metaanalyses also show an increased ovarian cancer risk in HRT users. The observed increase in risk was associated with ET and EPT. Among current users, an increased risk was observed after a duration of less than 5 years. (S3-Leitlinie Peri 2020) Regarding endometrial cancer, ET is associated with an increase in risk in non-hysterectomized women. Short-term EPT up to 
5 years of use does not seem to have a significant impact. (Beral et al. 2005) However, long-term EPT with a duration of use of more than 10 years can cause an increase in endometrial cancer risk. (Razavi et al. 2010) Therefore, ET should be used only in hysterectomized women (S3-Leitlinie Peri 2020).

The increase in cancer risk might be especially relevant for high-risk subgroups. According to recent prospective data, the cumulative risk at the age of 80 years in $B R C A 1$ mutation carriers is up to $72 \%$ for breast cancer and up to $44 \%$ for ovarian cancer. In BRCA2 mutation carriers, the cumulative breast cancer risk at the age of 80 years according to these data is increased up to $69 \%$ and for ovarian cancer up to $17 \%$, respectively (Kuchenbaecker et al. 2017). In a high-risk subgroup, possible risk modifiers are, therefore, to be used with caution. Several retrospective studies suggested that risk-reducing bilateral salpingo-oophorectomy (RRBSO) is associated with reduction in risk not only for ovarian and fallopian tube cancer, but also for breast cancer in BRCA1/2 mutation carriers (Rebbeck et al. 2009; Domchek et al. 2010). However, risk reduction for breast cancer after RRBSO in BRCA1/2 mutation carriers may have been overestimated because of selection bias in the existing observational studies (Heemskerk-Gerritsen et al. 2015). Prospective studies could not confirm a risk reduction for breast cancer especially in BRCAl mutation carriers (Mavaddat et al. 2020). In this study, we analyze the published data on HRT and risk for breast or ovarian cancer in $B R C A 1 / 2$ mutation carriers with and without RRBSO.

\section{Methods}

A search for relevant articles was run from January 1995 to October 2020 on PubMed. The MeSH used for the search was: "hormone replacement therapy", "BRCA mutation", "hormone replacement therapy", "breast cancer" or "ovarian cancer". The search created 70, 4548 and 877 hits, respectively. We also conducted a search within the Cochrane library. There were no Cochrane reviews available on HRT and risk of cancer in BRCA1/2 mutation carriers. The hits were searched for relevance, clinical trials, reviews, and metaanalyses. The authors of this review defined all articles as relevant which deal with the specific subject of whether or not HRT has an impact on breast and/or ovarian cancer in BRCA1/2 mutation carriers.

We found 11 relevant publications, 2 of which regarding ovarian cancer, 8 regarding breast cancer and 1 regarding endometrial cancer risk. 6 of the publications which investigated breast cancer risk focused on BRCA1/2 mutation carriers who had undergone RRBSO. After excluding crossmatch, there remained a total of nine publications to include in our systematic review. We included one case-control and one retrospective cohort study on HRT and ovarian cancer risk as well as one case-control study on HRT and breast cancer risk in BRCA1/2 mutation carriers with and without RRBSO. Furthermore, we included one case-control study, one Markov chain decision model, two prospective cohort studies and one metaanaylsis which examined the association between HRT and breast cancer risk in women with BRCA1/2 who had undergone RRBSO. Also, we included one case-control study on HRT-induced endometrial cancer risk in $B R C A 1 / 2$ mutation carriers in our review.

\section{Results}

\section{Ovarian cancer}

We found two adequate studies on the association between HRT and ovarian cancer risk in BRCA1/2 mutation carriers to include in our review (Table 1).

A case-control study by Kotsopoulos et al. from 2006 found no increase in ovarian cancer risk associated with use of HRT in BRCAl/2 mutation carriers $(\mathrm{OR}=0.93 ; 95 \% \mathrm{CI}$ $0.56-1.56 ; P=0.79$ ) (Kotsopoulos et al. 2006). The results were similar when tested separately for $B R C A 1$ ( $\mathrm{OR}=0.92$; 95\% CI 0.50-1.70; $P=0.80$ ) and $B R C A 2$ mutation carriers $(\mathrm{OR}=0.89 ; 95 \%$ CI $0.29-2.39 ; P=0.74)$. They included 117 cases and 256 controls with $B R C A 1$ as well as 45 cases and 119 controls with $B R C A 2$ mutation. All women had intact ovaries and underwent natural menopause. The mean age at enrollment was 62.7 years for cases and 61.2 years for controls $(P=0.51)$. A total of $45(27.8 \%)$ cases and $83(22.1 \%)$ controls reported ever use of HRT $(P=0.33)$. The average duration of HRT use was 5.4 years for cases and 7.6 years for controls, the difference was statistically significant $(P=0.05) .31$ of the cases and 65 of the controls reported ever use of estrogen-containing HRT, and 19 of the cases and 48 of the controls progestin-based HRT. No significant association was found with ovarian cancer risk and duration of HRT use (BRCA1: $P=0.14 ; B R C A 2: P=0.22$ ). When the type of HRT was examined, the study found a modest increase in ovarian cancer risk associated with ever use of estrogen $(\mathrm{OR}=1.5 ; 95 \%$ CI $0.73-3,11)$ and a decrease in risk associated with ever use of progestin $(\mathrm{OR}=0.57 ; 95 \%$ CI 0.24-1.35) compared with never use of HRT. However, both effects were not significant. Use of estrogen was defined as use of either estrogen or estrogen and progestin-based HRT, use of progestin as use of either progestin or estrogen and progestin-based HRT. The effects associated with different formulations of HRT were not investigated separately for BRCA1 and BRCA2 mutation carriers.

Perri et al. included a total of $718 B R C A 1$ and $331 B R C A 2$ mutation carriers to conduct a retrospective cohort study (Perri et al. 2015). The main purpose of the study was to 
investigate the association between ovarian cancer and fertility treatments. Also, the study investigated potential confounding effects including use of HRT. Multivariate analysis showed an increase in ovarian cancer risk associated with use of HRT for $B R C A 1 / 2$ mutation carriers $(\mathrm{OR}=1.98$; 95\% CI 1.21-3.25). Analysis was performed separately for $B R C A 1(\mathrm{OR}=1.66 ; 95 \% \mathrm{CI} 0.89-3.08 ; P<0.001)$ and $B R C A 2(\mathrm{OR}=3.04 ; 95 \%$ CI 1.19-7.8; $P<0.001)$. However, the separate analysis reduced the significance of the reported association for BRCA1 mutation carriers. 32 of the cases, who were defined by history of IVF, and 73 of the controls reported use of HRT, while 136 of the cases and 800 of the controls declared no use. Surveillance of cases and controls ended at RRBSO, which was performed on a total of 114 participants. All of the participants were Jewish Israeli women with personal or family history of BRCA mutationassociated cancers, leading to an overrepresentation of a specific ethnic group. Numbers of cases and controls who used HRT were low and there was no information provided on duration of use and different formulations.

\section{Breast cancer}

Regarding breast cancer, we found one study on the association between HRT and risk of cancer in mutation carriers with and without RRBSO. All of them included BRCAl mutation carriers, only. Furthermore, we identified data from five publications on breast cancer risk associated with HRT use in BRCA1/2 mutation carriers undergoing RRBSO (Table 2).

\section{BRCA1 mutation carriers with and without RRBSO}

Kotsopoulos et al. included 432 matched pairs with BRCAl mutation in their case-control study from 2016 (Kotsopoulos et al. 2016). A number of the included participants overlap with a prospective cohort study conducted by Rebbeck et al. in 2005, which will be discussed below (Rebbeck et al. 2005).

Also, the case-control study by Kotsopoulos et al. is an extension to an earlier report by Eisen et al. published in 2008, which included 236 matched case-control pairs with BRCA1 mutation (Eisen et al. 2008). For ever compared to never use of HRT, a decrease in breast cancer risk was observed (OR $=0.58 ; 95 \%$ CI $0.35-0.96 ; P=0.03)$. When different formulations of HRT were examined, Eisen et al. found a significant risk reduction associated with use of ET $(\mathrm{OR}=0.51 ; 95 \%$ CI $0.27-0.98 ; P=0.04)$ and a non-significant risk reduction associated with $\mathrm{EPT}(\mathrm{OR}=0.66 ; 95 \% \mathrm{CI}$ $0.34-1.27 ; P=0.21)$. Information on estrogen receptor (ER) status was available in 103 of the cases (44\%). In $12 \%$ of the cases with ER-positive and $23 \%$ with ER-negative breast cancer, HRT was reported. Eisen et al. concluded that HRT might not be contraindicated for BRCAl mutation carriers.

However, multivariate analysis in the extension study by Kotsopoulos et al. found no association between breast cancer risk and ever compared to never use of $\mathrm{HRT}(\mathrm{OR}=0.80$; 95\% CI 0.55-1.16; $P=0.24)$. Also, no association with different HRT formulations was observed $(P \geq 0.11) .46$ of the cases and 42 of the controls reported ET use. 28 of the cases and 41 of the controls used EPT, while 2 cases and 3 controls reported progesterone monotherapy.

The type of menopause was natural in 327 (75.7\%) of the cases and controls, and surgical in the remaining participants. Women after bilateral mastectomy were excluded from the study. The mean age at enrollment was 58 years for cases and 58.3 for controls. $18.5 \%$ of the cases and $21.3 \%$ of the controls reported ever use of HRT $(P=0.31)$. The mean duration of use was 4.42 years for cases and 4.27 for controls $(P=0.83)$. Analysis was carried out separately for surgical and natural menopause. The odds ratio for women with surgical menopause was higher $(\mathrm{OR}=1.06$; $95 \%$ CI $0.58-1.96$; $P=0.85$ ) than for those with natural menopause (OR 0.72; $95 \%$ CI $0.44-1.18 ; P=0.20$ ), although the difference was not statistically significant. The results did not differ by duration ( $\geq 3$ years and $<3$ years) or recency of use. Furthermore, no association was observed with age at menopause $(\leq 45$ years of age or more) and breast cancer diagnosis ( $\leq 50$ years of age or more).

\section{BRCA1/2 mutation carriers after RRBSO}

Rebbeck et al. included a total of 122 BRCA1 mutation carriers in their matched case--control study from 1999 (Rebbeck et al. 1999). They included 43 healthy carriers with RRBSO and 79 controls without surgery to examine the breast cancer risk after RRBSO. The study found a significant reduction in breast cancer risk associated with RRBSO in the total sample $(\mathrm{HR}=0.53 ; 95 \%$ CI $0.33-0.84)$. When women with HRT exposure were excluded from the analysis $(n=22)$, a further decrease in risk was observed $(\mathrm{HR}=0.42$; $95 \% \mathrm{CI}=0.22-0.81$ ). As the HR estimate in the total sample was only marginally higher, the authors assumed no more than a moderate increase in breast cancer risk associated with HRT after prophylactic surgery. They conclude, that HRT use does not negate the reduction in breast cancer risk associated with RRBSO. The mean age of the healthy carriers at the time of surgery was 39.4 years, the mean corresponding age of the controls was 35.3. Women who had undergone mastectomy prior to BSO were excluded. The cases were followed up for an average of 9.6 years after surgery, the controls for an average of 8.1 years. $69 \%$ of the cases and $6 \%$ of the controls reported ever use of HRT. The sample size of the study was small and there was no 
Table 1 HRT and risk of ovarian cancer in BRCA mutation carriers

\begin{tabular}{|c|c|c|}
\hline $\begin{array}{l}\text { Study/study design/Oxford center of evidence } \\
\text { based medicine (OCEBM) level of evidence } \\
\text { (LOE) }\end{array}$ & Number & Results \\
\hline $\begin{array}{l}\text { Kotsopoulos et al. (2006) } \\
\text { Case-control study } \\
\text { LOE } 3 b\end{array}$ & $\begin{array}{l}\text { Cases: } \\
117 \text { BRCA1, } 45 \text { BRCA2 } \\
\text { Controls: } \\
256 \text { BRCA1, } 119 \text { BRCA2 }\end{array}$ & $\begin{array}{l}\text { No increase in risk overall } \\
B R C A 1: \mathrm{OR}=0.92 ; 95 \% \text { CI } 0.50-1.70 ; P=0.80 \\
B R C A 2: \mathrm{OR}=0.89 ; 95 \% \text { CI } 0.29-2.39 ; P=0.74 \\
\text { Non-significant increase in risk associated with ever use of } \\
\text { estrogen }(\mathrm{OR}=1.5 ; 95 \% \text { CI } 0.73-3.11) \text {, non-significant } \\
\text { decrease in risk associated with ever use of progestin } \\
(\mathrm{OR}=0.57 ; 95 \% \text { CI } 0.24-1.35) \\
\text { No association with duration of use }\end{array}$ \\
\hline $\begin{array}{l}\text { Perri et al. (2015) } \\
\text { Retrospective cohort study } \\
\text { LOE } 2 b\end{array}$ & $\begin{array}{l}718 \text { BRCA1, } 331 \text { BRCA2 } \\
\text { (use of HRT in total: 105) }\end{array}$ & $\begin{array}{l}\text { Increase in risk } \\
B R C A 1: \text { OR }=1 . \\
66 ; 95 \% \text { CI } 0.89-3.08 ; P<0.001 \\
B R C A 2: \text { OR }=3.04 ; 95 \% \text { CI } 1.19-7.8 ; P<0.001\end{array}$ \\
\hline
\end{tabular}

complete information on the menopausal status of the participants. Information on timing, duration, dose, and different formulations of HRT was not provided and data on HRT use were not complete.

Armstrong et al. used a Markov decision analytic model to investigate the impact of HRT after RRBSO in premenopausal BRCA1/2 mutation carriers (Armstrong et al. 2004). HRT was defined as combined EPT. A gain in life expectancy associated with RRBSO between the age of 30 and 40 years was found, irrespective of whether or not HRT was used. The decrease of ovarian and cancer risk outweighed the increase in risk of cardiovascular diseases and osteoporosis. Additional prophylactic bilateral mastectomy was associated with a further increase in life expectancy.

For $B R C A 1 / 2$ mutation carriers without bilateral mastectomy, HRT from the time of RRBSO until the age of 50 years was associated with relatively small changes in life expectancy (ranging from -0.34 years for 30 -year-old women to +0.17 years for 40-year-old women). When HRT was continued for life, a decrease in life expectancy was observed (ranging from -1.09 to -0.76 years, respectively).

Use of HRT until the age of 50 years after bilateral mastectomy and oophorectomy resulted in a gain in life expectancy (ranging from 0.78 years for 30-year-old women to 0.79 years for 40 -year-old women). When HRT was continued for life, the gain in life expectancy in this subgroup dropped to an amount of 0.39 and 0.37 years.

The PROSE study group around Rebbeck et al. included 462 BRCA1/2 mutation carriers for a prospective cohort study in 2005 (Rebbeck et al. 2005). The authors report a partial overlap with their publication from 1999, which has been discussed previously (Rebbeck et al. 1999). However, the authors emphasize the recruitment of additional participants and different inclusion and exclusion criteria for the two studies. In the study by Rebbeck et al., HRT exposure had no impact on the reduction of breast cancer risk associated with RRBSO in $B R C A 1 / 2$ mutation carriers $(\mathrm{HR}=0.37 ; 95 \% \mathrm{CI} 0.14-0.96)$ compared to the entire cohort. 155 of the included women underwent RRBSO. The majority, $71 \%$ of the included women with and $67 \%$ of those without RRBSO, had a BRCA1 mutation. Bilateral mastectomy led to an exclusion from the study, as well as a history of ovarian or breast cancer before or within 6 months after recruitment. 12 of the $B R C A 1 / 2$ mutation carriers with and 65 without RRBSO were diagnosed with breast cancer during follow-up. Follow-up was 3.6 years after RRBSO. The mean age at RRBSO was 42.7 years. The mean age at breast cancer diagnosis was 45.6 for the cases with and 39.3 for the controls without RRBSO. Different formulations of HRT were also examined. No difference was found, although the sample sizes were relatively small with 54 women who took ET and 34 who took EPT, respectively. Separate analyses for BRCA1 and BRCA2 mutation carriers were not conducted and an association with the duration of use was not examined.

In 2011, Domchek et al. published data on an extension and follow-up to this study (Domchek et al. 2011). In total, 795 BRCA1 and 504 BRCA2 mutation carriers were included. 321 women underwent RRBSO, of whom $45 \%$ used HRT after surgery. Mean follow-up was 5.4 years after RRBSO. Like in the initial study, HRT following RRBSO was not associated with an increase in breast cancer risk. When different types of HRT were examined, neither ET nor EPT led to an increase in risk. For BRCAl mutation carriers, a decrease in breast cancer risk associated with HRT was found for both women with $(\mathrm{HR}=0.52 ; 95 \%$ CI $0.30-0.92)$ and without RRBSO (HR $=0.29$; 95\% CI 0.13-0.69).

Kotsopoulos et al. conducted a prospective cohort study in 2018 which included 872 BRCA1 mutation carriers undergoing RRBSO (Kotsopoulos et al. 2018). A subset overlapped with the previously performed case-control study by the same authors (Kotsopoulos et al. 2016). The primary 


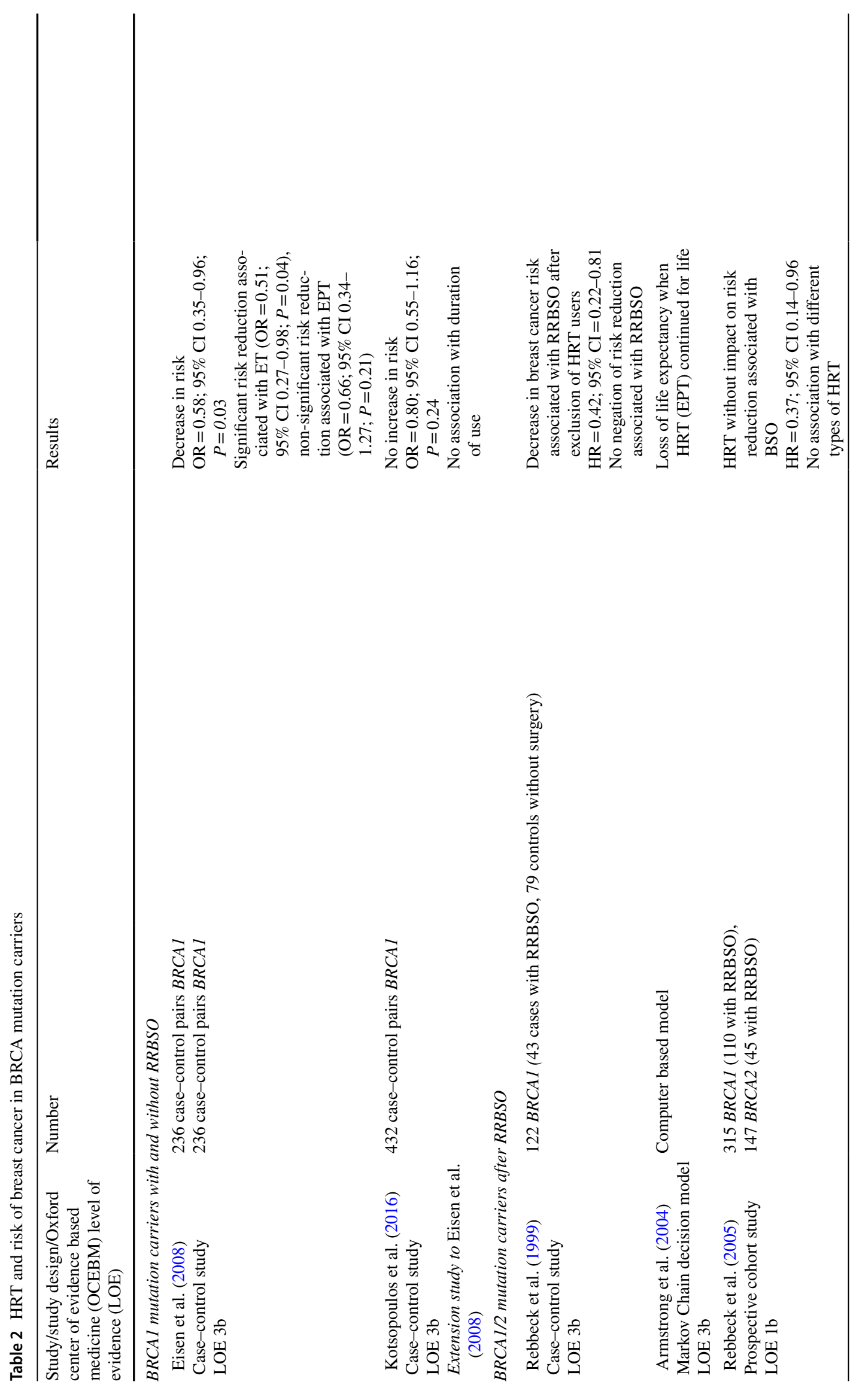




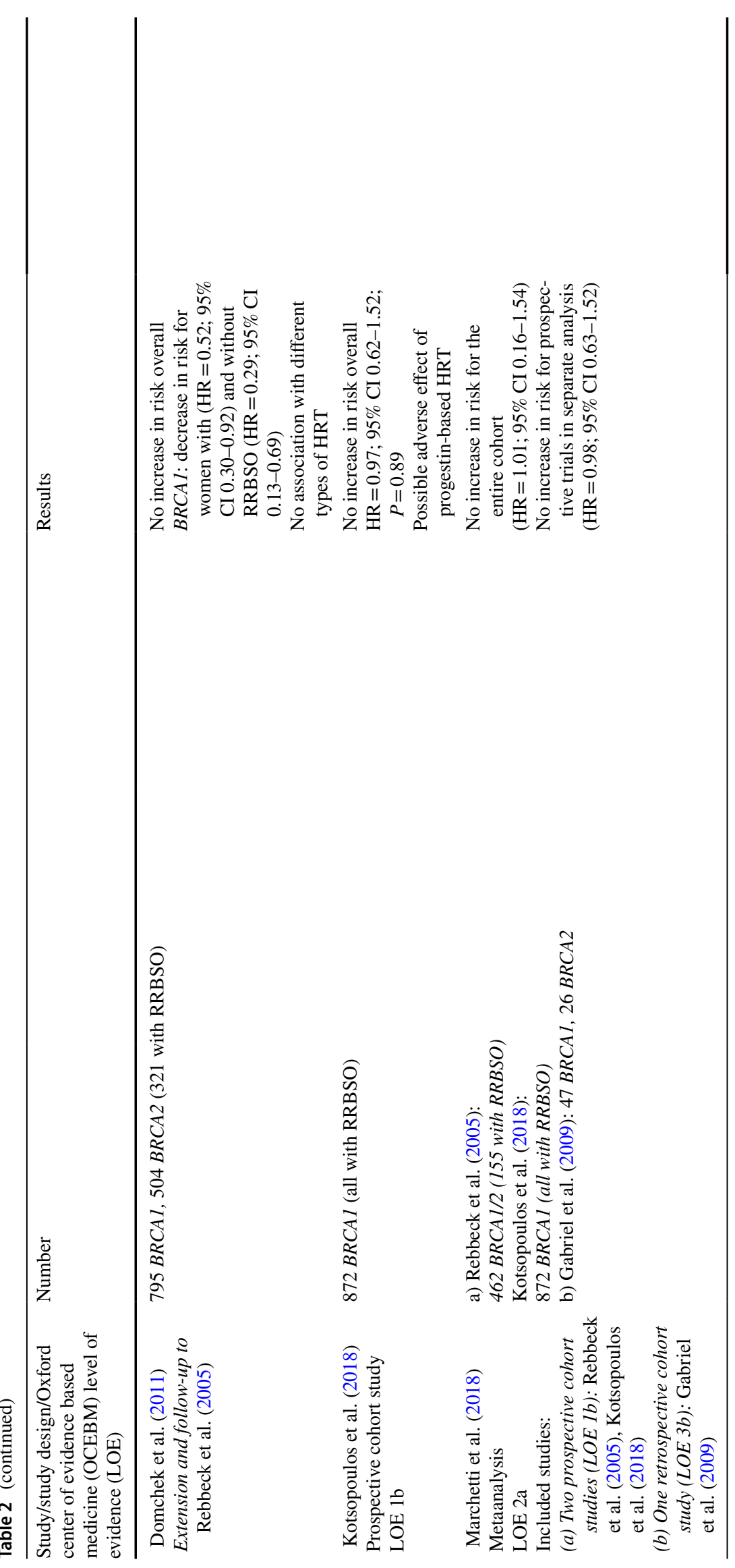


Table 3 HRT and risk of endometrial cancer in BRCA mutation carriers

\begin{tabular}{lll}
\hline $\begin{array}{l}\text { Study/study design/Oxford Center of Evidence based Medi- } \\
\text { cine (OCEBM) level of evidence (LOE) }\end{array}$ & Number & Results \\
\hline Segev et al. (2015) & Cases: & No increase in risk overall \\
Case-control study & 62 BRCA1, 21 BRCA2 & OR $=0.73 ; 95 \%$ CI $0.33-1.63 ; P=0.44$ \\
LOE 3b & Controls: & Increase in risk associated with progesterone-only \\
& $951 B R C A 1,76$ BRCA1 & $($ OR $=6.91 ; 95 \%$ CI $0.99-98.1 ; P=0.05)$, non-sig- \\
& & nificant decrease in risk associated with estrogen- \\
& & only (OR $=0.23 ; 95 \%$ CI $0.03-1.78 ; P=0.16)$ \\
\hline
\end{tabular}

endpoint was incident invasive breast cancer, which was diagnosed in $92(10.6 \%)$ of the included carriers. Women with a prior diagnosis of breast cancer or with a history of bilateral mastectomy were excluded from the study. The mean range between RRBSO and breast cancer diagnosis was 4.5 years.

The results did not show an association between breast cancer risk and HRT after RRBSO $(\mathrm{HR}=0.97 ; 95 \% \mathrm{CI}$ $0.62-1.52 ; P=0.89)$. However, differing effects associated with different types of HRT were observed, suggesting a possible adverse effect of progestin-based HRT. 377 (43\%) women used HRT after oophorectomy and 495 (57\%) did not. The mean duration of HRT use was 3.9 years. Women who used HRT had a mean age of 40.3 years at enrollment, women who did not a mean age of 45.8. The mean age at RRBSO was 43.0 and 48.4 years, respectively $(P<0.0001)$. Mean follow-up duration was 7.9 years for women who used HRT and 7.4 years for women who did not $(P<0.07)$. Among the HRT users, 259 (69\%) used ET, 66 (18\%) EPT, and $40(11 \%)$ used progesterone monotherapy. The remaining $21 \%$ used other formulations. After 10 years of followup, the cumulative incidence of breast cancer was significantly lower for BRCA1/2 mutation carriers who used ET (12\%) compared to those who used EPT $(22 \% ; P=0.04)$. The observed effect was even more pronounced for women prior to the age of 45 years ( $9 \%$ and $24 \%$, respectively; $P=0.009$ ).

Marchetti et al. included two prospective and one retrospective cohort studies in their metaanalysis published in 2018 (Marchetti et al. 2018). In total, the three studies comprised 1100 BRCA1/2 mutation carriers after RRBSO (Rebbeck et al. 2005; Kotsopoulos et al. 2018; Gabriel et al. 2009).

The two prospective cohort studies by Rebbeck et al. and Kotsopoulos et al. have already been described above (Rebbeck et al. 2005; Kotsopoulos et al. 2018). In the retrospective cohort study by Gabriel et al. 47 BRCA1 and 26 BRCA2 mutation carriers were included (Gabriel et al. 2009). The main aim of this study was to investigate differences in HRT use associated with and without total abdominal hysterectomy at the time of RRBSO in BRCA1/2 mutation carriers. History of breast cancer led to exclusion of the study. 55\% of the included women underwent hysterectomy in addition to RRBSO. 17 of the hysterectomized and 16 of the nonhysterectomized women used HRT and $50 \%$ of the HRT users had a prophylactic mastectomy. 3 of 17 women who used ET and 9 of 29 women who did not use HRT developed breast cancer. Although HRT use was not associated with more breast cancer cases, no data on estimated breast cancer risk were given and no further analysis was performed.

In the metaanalysis, no increase in breast cancer risk associated with HRT was found for the entire cohort $(\mathrm{HR}=1.01$; 95\% CI 0.16-1.54). Metaanalysis was also performed separately for the two prospective trials, but no negative impact on breast cancer risk was found $(\mathrm{HR}=0.98 ; 95 \%$ CI 0.63-1.52). The mean duration of HRT was 3.3 years. Different HRT formulations were also examined. 326 of included women used ET and 114 used EPT. No increase in risk was found associated with the different types of HRT. However, breast cancer risk associated with ET use was lower compared to use of EPT, both in the entire cohort $(\mathrm{OR}=0.62 ; 95 \% \mathrm{CI} 0.29-1.31)$ and in the prospective studies only $(\mathrm{OR}=0.62 ; 95 \%$ CI $0.29-1.31)$.

Marchetti et al. conclude, that HRT following RRBSO in BRCA1/2 mutation carriers seems to be a safe therapeutic option. However, they point out the low number especially of BRCA2 mutation carriers and the need of larger prospective and randomized studies in the future.

\section{Endometrial cancer}

Segev et al. included 62 cases and 951 controls with BRCA1 and 21 cases and 76 controls with $B R C A 2$ mutation in their case-control study (Segev et al. 2015) (Table 3). No overall association between HRT and endometrial cancer was found $(\mathrm{OR}=0.73 ; 95 \% \mathrm{CI} 0.33-1.63 ; P=0.44)$. However, an increase in risk for endometrial cancer associated with progestin-treatment $(\mathrm{OR}=6.91 ; 95 \%$ CI $0.99-98.1 ; P=0.05)$ and a non-significant decrease in risk associated with ET (OR $=0.23 ; 95 \%$ CI $0.03-1.78 ; P=0.16)$ were observed. The separate analysis for $B R C A 1$ and $B R C A 2$ mutation carriers did not show an overall association between HRT and endometrial cancer, either. For $B R C A 1$ mutation carriers, multivariate analysis showed a non-significant risk reduction associated with ET $(\mathrm{OR}=0.52 ; 95 \%$ CI $0.06-4.23$; $P=0.54)$ a non-significant increase in risk associated with 
EPT $(\mathrm{OR}=1.77 ; 95 \%$ CI $0.20-2.98 ; P=0.70)$. Progestinonly HRT was associated with a significant increase in endometrial cancer risk $(\mathrm{OR}=16.5$; 95\% CI 2.02-134 $P=0.009)$ in BRCA1 mutation carriers. In BRCA2 mutation carriers, EPT was associated with a decrease in risk $(\mathrm{OR}=0.35 ; 95 \%$ CI $0.02-5.15 ; P=0.44)$. Due to small numbers, no analyses could be conducted for ET and progestin therapy in $B R C A 2$ mutation carriers. The mean age at baseline was 55.5 years for cases and 56.1 for controls $(P=0.65) .28 .9 \%$ of the cases and $41.9 \%$ of the controls were premenopausal, compared to $71.1 \%$ and $58.1 \%$ who were postmenopausal $(P=0.02) .13$ $(16.1 \%)$ of the cases and $157(15.9 \%)$ the controls reported use of HRT $(P=0.96) .2$ of the cases and 42 of the controls took ET, 8 and 63 took EPT, respectively. 1 of the cases and 48 of the controls reported use of other formulations or type of HRT was unknown. Numbers and exposure to HRT were small: only 13 of the cases used HRT, 2 of which took ET. No information was given on duration and timing of HRT.

\section{Discussion}

For ovarian cancer, one of the studies showed no overall association between HRT and cancer risk in BRCA1/2 mutation carriers (Kotsopoulos et al. 2006). However, an increase in risk associated with estrogen- and a decrease in risk associated with progestin-containing HRT was observed, each being non-significant. The second study which primarily investigated the effect of fertility treatments on ovarian cancer reported an increase in risk associated with HRT (Perri et al. 2015). However, numbers of women in this study were low and data on different formulations of HRT were lacking.

For breast cancer, most of the studies including BRCAI mutation carriers with and without RRBSO did not find an increase in risk associated with HRT, neither for woman after surgical, nor after natural menopause (Kotsopoulos et al. 2016; Eisen et al. 2008). One of the studies even found a significant reduction of breast cancer risk associated with ever use of HRT as well as with ET and a non-significant risk reduction associated with EPT (Eisen et al. 2008). However, the later extension to this study by Kotsopoulos et al. did not confirm the finding of a reduction in risk (Kotsopoulos et al. 2016).

The studies investigating an association between HRT and breast cancer risk in BRCAl/2 mutation carriers after RRBSO mostly did not find an adverse effect, either (Rebbeck et al. 2005; Kotsopoulos et al. 2018; Marchetti et al. 2018). One case-control study by Rebbeck et al. could not exclude a moderate increase in breast cancer risk associated with HRT, although the authors concluded that the reduction in breast cancer risk associated with RRBSO was not abolished by the use of HRT (Rebbeck et al. 1999). A subsequent prospective study by the same authors found no impact on the reduction of breast cancer risk associated with RRBSO in BRCA1/2 mutation carriers (Rebbeck et al. 2005). The extension and follow-up data from 2011 even reported a decrease in breast cancer risk associated with HRT in BRCAl mutation carriers with and without RRBSO (Domchek et al. 2011). In contrast, Armstrong et al. postulated an adverse effect on life expectancy in their Markov Chain decision model, when HRT was continued for life after risk-reducing surgery (Armstrong et al. 2004). However, HRT was defined as EPT and no other formulations were examined. No relevant effect was found when HRT was stopped at the age of 50 years. The analytic model was based on analyses of the effects of HRT among women with a family history of breast cancer, not specifically on BRCAl/2 mutation carriers, where HRT might have a different effect. Also, the analytic model from 2004 might not contain current assumptions and may be outdated. Therefore, it might not be appropriate for current clinical decision making.

Regarding different types of HRT, the prospective study by Rebbeck et al. as well as its extension by Domcheck et al. did not find an association with breast cancer risk (Rebbeck et al. 2005; Domchek et al. 2011). Another prospective cohort study by Kotsopoulos et al. observed a significantly lower cumulative incidence of breast cancer in BRCA1/2 mutation carriers who used ET compared to EPT after RRBSO after 10 years of follow-up, suggesting a possible adverse effect of progestin-based HRT (Kotsopoulos et al. 2018). These two study groups were the first to contribute prospective data to the discussion about HRT in BRCA mutation carriers, focusing exclusively on mutations in BRCA1. In the metaanalysis by Marchetti et al., another smaller retrospective study was additionally included and a lower breast cancer risk associated with the use of ET compared to EPT was observed (Marchetti et al. 2018). However, no actual increase in risk associated with the different types of HRT was found.

Recently, the safety of endocrine interventions in BRCA1/2 mutation carriers has gradually gained attention. A number of systematic reviews addressing breast cancer risk associated with HRT following RRBSO in BRCA1/2 mutation carriers have been published (Siyam et al. 2017; Birrer et al. 2018; Gordhandas et al. 2019; Vermeulen et al. 2019; Gaba and Manchanda 2020). According to one of those reviews, the safety of HRT is demonstrated best in the PROSE study by Rebbeck in 2005 (Birrer et al. 2018). In contrast, another review points out the small number of women who took EPT in this study and concludes that safety of HRT types other than ET cannot be definitely evaluated based on these data (Gordhandas et al. 2019). In general, most of the reviewers conclude, that there is no evidence for an increase in breast cancer risk in BRCAl/2 mutation carriers after RRBSO, but that more data are needed to draw clear conclusions. Vermeulen et al. especially point out the 
possible adverse effect of progestin-containing HRT found by Kotsopoulos et al. and the need of further investigation in this direction (Kotsopoulos et al. 2018; Vermeulen et al. 2019). In contrast, the recently published review by Gaba et al. did not find a change in clinical practice justifiable based on this study due to small numbers and inconsistent follow-up (Gaba and Manchanda 2020). All of the reviews explicitly investigated the safety of HRT in BRCAl/2 mutation carriers after RRBSO. However, most of them also presented studies which included women with and without RRBSO (Birrer et al. 2018; Gordhandas et al. 2019; Gaba and Manchanda 2020). A clear separation and differentiated evaluation between these two subgroups was lacking.

Regarding endometrial cancer risk associated with HRT use in BRCA1/2 mutation carriers, we found only one publication (Segev et al. 2015). In contrast to the general population, an increase in risk associated with progesterone monotherapy and a non-significant decrease in risk associated with estrogen monotherapy was observed. When the analysis was performed separately for $B R C A 1$, the described effect was confirmed and a non-significant increase in risk associated with EPT was seen. In contrast, a decrease in risk associated with EPT was observed in BRCA2 mutation carriers. However, numbers especially of cancer cases and exposure to HRT were small. There were only two BRCAI mutation carriers diagnosed with endometrial cancer who were exposed to ET and analyses associated with monotherapy could not be performed in BRCA2 mutation.

Overall, data on cancer risk associated with HRT use in $B R C A 1 / 2$ mutation carriers are limited. Sample sizes were often small, especially regarding $B R C A 2$ mutation carriers which were included in only few studies (Kotsopoulos et al. 2006; Perri et al. 2015; Rebbeck et al. 2005; Gabriel et al. 2009). In addition, the majority of these studies did not perform subgroup analyses for BRCA1 and BRCA2 (Kotsopoulos et al. 2006; Rebbeck et al. 2005; Gabriel et al. 2009). Between some of the studies regarding breast cancer risk, a partial overlap was reported (Kotsopoulos et al. 2016; Rebbeck et al. 2005; Eisen et al. 2008; Domchek et al. 2011). Although most of the included studies in this review examined the impact of different HRT formulations, none of them took different dosages or treatment routines, such as sequential or continuous combined HRT, into consideration.

Due to elevated risk of cancer, $B R C A 1 / 2$ mutation carriers are increasingly encouraged to undergo RRBSO after completion of childbearing (Birrer et al. 2018; Domchek and Kaunitz 2016). However, there are studies suggesting that early menopause and subsequent estrogen loss does not only cause a decrease in quality of life due to menopausal symptoms, but may also increase cardiovascular diseases, osteoporosis, and cognitive dysfunction (Parker et al. 2009; Georgakis et al. 2019). Also, there are indications that BSO is associated with an increased mortality in the general population, especially when BSO is performed at a younger age of 45-50 years and no HRT is used (Parker et al. 2009; Rocca et al. 2006). In contrast, large prospective studies observed a decrease in all-cause mortality associated with RRBSO in BRCA1/2 mutation carriers compared to no RRBSO. If HRT until the age of natural menopause could increase survival further has not been analyzed so far. However, there are data supporting an effect of HRT after RRBSO in BRCA1/2 mutation carriers on menopausal symptoms such as hot flashes (Madalinska et al. 2006). Although the Women's Health Initiative, the largest study to date on peri- and postmenopausal HRT, did not find a protective cardiovascular effect associated with HRT, younger women might still benefit (Rossouw et al. 2007; Manson et al. 2007).

Although data are limited, HRT does not seem to be associated with relevant increases in breast cancer risk according to the available studies, especially for $B R C A 1 / 2$ mutation carriers who had RRBSO. Given the risks of RRBSO in younger premenopausal women, HRT can, therefore, be recommended after RRBSO prior to the age of 50 years. ET is associated with an increasing risk of endometrial cancer and, therefore, not recommended for non-hysterectomized women. But also long-term EPT can cause an increase in endometrial cancer risk. (Razavi et al. 2010) Risk for endometrial cancer in BRCA1/2 mutation carriers is being discussed controversially. There are some studies which suggest a higher risk of endometrial cancer in BRCAl/2 mutation carriers (Laitman et al. 2019). Segev et al. found a higher risk of endometrial cancer among BRCAl mutation carriers compared to the general population which, however, was largely attributable to the use of Tamoxifen (Segev et al. 2013). According to existing data, mutation in BRCAl/2 genes alone is not an indication for hysterectomy. Hysterectomy at the time of RRBSO, therefore, needs to be discussed individually depending on age and additional risk factors.

In conclusion, there is insufficient evidence on the effect of HRT on ovarian and endometrial cancer in BRCAl/2 mutation carriers. Also, data on the association of HRT use and breast cancer in $B R C A 1 / 2$ mutation carriers are limited, especially for $B R C A 2$ mutation carriers. More prospective studies with longer follow-up periods are needed to assess the safety and to further investigate different formulations, doses and regimen of HRT. Due to elevated cancer risk, RRBSO is recommended at the age of about 35-40 years in BRCA1 and 40-45 years in BRCA2 mutation carriers, whereby family planning and the earliest age at diagnosis in affected family members have to be taken into consideration (Wagner and Reuß 2019; The Australian Cancer Network and National Breast Cancer Centre 2004). However, as ovarian cancer can occur also before the age of 35 years and data on RRBSO in BRCA1/2 mutation carriers is still limited, the ideal timing of RRBSO remains difficult (Finch et al. 2014; Marchetti et al. 2014). According to limited data 
from available studies, HRT following RRBSO in BRCA1/2 mutation carriers does not seem to have an adverse effect or negate the reduction in breast cancer risk associated with RRBSO. Adequate HRT after RRBSO should be offered to premenopausal women to avoid postmenopausal symptoms and chronic diseases resulting from low estrogen levels such as osteoporosis and myocardial infarction. Regarding $B R C A 1 / 2$ mutation carriers who have not undergone RRBSO, HRT does not seem to have a relevant impact on cancer risk. Short-term HRT may be considered in BRCA1/2 mutation carriers without RRBSO to reduce perimenopausal symptoms. However, indication should be strict and patients should be informed about limited data on the safety concerning cancer risk. ET is recommended after hysterectomy but should be avoided in non-hysterectomized women due to possible increases in endometrial cancer risk.

Author contributions DHr: Literature search, data analysis, and manuscript writing. SS: Data analysis and manuscript writing. KK: Manuscript editing. GE: Manuscript editing. OO: Project development and manuscript editing.

Funding Open Access funding enabled and organized by Projekt DEAL. No funding was received for this work.

\section{Declarations}

Conflict of interest The authors declare that they have no conflict of interest.

Open Access This article is licensed under a Creative Commons Attribution 4.0 International License, which permits use, sharing, adaptation, distribution and reproduction in any medium or format, as long as you give appropriate credit to the original author(s) and the source, provide a link to the Creative Commons licence, and indicate if changes were made. The images or other third party material in this article are included in the article's Creative Commons licence, unless indicated otherwise in a credit line to the material. If material is not included in the article's Creative Commons licence and your intended use is not permitted by statutory regulation or exceeds the permitted use, you will need to obtain permission directly from the copyright holder. To view a copy of this licence, visit http://creativecommons.org/licenses/by/4.0/.

\section{References}

Armstrong K, Schwartz JS, Randall T, Rubin SC, Weber B (2004) Hormone replacement therapy and life expectancy after prophylactic oophorectomy in women with BRCA1/2 mutations: a decision analysis. J ClinOncol 22(6):1045-1054

Beral V, Bull D, Reeves G (2005) Endometrial cancer and hormone-replacement therapy in the million women study. Lancet 365(9470):1543-1551

Birrer N, Chinchilla C, Del Carmen M, Dizon DS (2018) Is hormone replacement therapy safe in women with a BRCA mutation?: A systematic review of the contemporary literature. Am J ClinOncol 41(3):313-315
Collaborative Group on Hormonal Factors in Breast Cancer (2019) Type and timing of menopausal hormone therapy and breast cancer risk: individual participant meta-analysis of the worldwide epidemiological evidence. Lancet 394(10204):1159-1168

Domchek S, Kaunitz AM (2016) Use of systemic hormone therapy in BRCA mutation carriers. Menopause 23(9):1026-1027

Domchek SM, Friebel TM, Singer CF, Evans DG, Lynch HT, Isaacs $\mathrm{C}$ et al (2010) Association of risk-reducing surgery in BRCA1 or BRCA2 mutation carriers with cancer risk and mortality. JAMA 304(9):967-975

Domchek SM, Friebel T, Neuhausen SL, Lynch HT, Singer CF, Eeles RA et al (2011) Is hormone replacement therapy (HRT) following risk-reducing salpingo-oophorectomy (RRSO) in BRCA1 (B1)and BRCA2 (B2)-mutation carriers associated with an increased risk of breast cancer? J ClinOncol 29(15_suppl):1501

Eisen A, Lubinski J, Gronwald J, Moller P, Lynch HT, Klijn J et al (2008) Hormone therapy and the risk of breast cancer in BRCA1 mutation carriers. J Natl Cancer Inst 100(19):1361-1367

Finch APM, Lubinski J, Møller P, Singer CF, Karlan B, Senter L et al (2014) Impact of oophorectomy on cancer incidence and mortality in women with a BRCA1 or BRCA2 mutation. J ClinOncol 32(15):1547-1553

Gaba F, Manchanda R (2020) Systematic review of acceptability, cardiovascular, neurological, bone health and HRT outcomes following risk reducing surgery in BRCA carriers. Best Pract Res ClinObstetGynaecol 65:46-65

Gabriel CA, Tigges-Cardwell J, Stopfer J, Erlichman J, Nathanson K, Domchek SM (2009) Use of total abdominal hysterectomy and hormone replacement therapy in BRCA1 and BRCA2 mutation carriers undergoing risk-reducing salpingo-oophorectomy. Fam Cancer 8(1):23-28

Georgakis MK, Beskou-Kontou T, Theodoridis I, Skalkidou A, Petridou ET (2019) Surgical menopause in association with cognitive function and risk of dementia: a systematic review and metaanalysis. Psychoneuroendocrinology 106:9-19

Gordhandas S, Norquist BM, Pennington KP, Yung RL, Laya MB, Swisher EM (2019) Hormone replacement therapy after risk reducing salpingo-oophorectomy in patients with BRCA1 or BRCA2 mutations; a systematic review of risks and benefits. GynecolOncol 153(1):192-200

Heemskerk-Gerritsen BAM, Seynaeve C, van Asperen CJ, Ausems MGEM, Collée JM, van Doorn HC et al (2015) Breast cancer risk after salpingo-oophorectomy in healthy BRCA1/2 mutation carriers: revisiting the evidence for risk reduction. J Natl Cancer Inst 107(5):djv033

Kotsopoulos J, Lubinski J, Neuhausen SL, Lynch HT, Rosen B, Ainsworth $\mathrm{P}$ et al (2006) Hormone replacement therapy and the risk of ovarian cancer in BRCA1 and BRCA2 mutation carriers. Gyneco1Oncol 100(1):83-88

Kotsopoulos J, Huzarski T, Gronwald J, Moller P, Lynch HT, Neuhausen SL et al (2016) Hormone replacement therapy after menopause and risk of breast cancer in BRCA1 mutation carriers: a case-control study. Breast Cancer Res Treat 155(2):365-373

Kotsopoulos J, Gronwald J, Karlan BY, Huzarski T, Tung N, Moller $P$ et al (2018) Hormone replacement therapy after oophorectomy and breast cancer risk among BRCA1 mutation carriers. JAMA Oncol 4(8):1059-1065

Kuchenbaecker KB, Hopper JL, Barnes DR, Phillips K-A, Mooij TM, Roos-Blom M-J et al (2017) Risks of breast, ovarian, and contralateral breast cancer for BRCA1 and BRCA2 mutation carriers. JAMA 317(23):2402-2416

Laitman Y, Michaelson-Cohen R, Levi E, Chen-Shtoyerman R, Reish O, Josefsberg Ben-Yehoshua S et al (2019) Uterine cancer in Jewish Israeli BRCA1/2 mutation carriers. Cancer 125(5):698-703

Madalinska JB, van Beurden M, Bleiker EMA, Valdimarsdottir HB, Hollenstein J, Massuger LF et al (2006) The impact of hormone 
replacement therapy on menopausal symptoms in younger highrisk women after prophylactic salpingo-oophorectomy. J ClinOncol 24(22):3576-3582

Manson JE, Allison MA, Rossouw JE, Carr JJ, Langer RD, Hsia J et al (2007) Estrogen therapy and coronary-artery calcification. N Engl J Med 356(25):2591-2602

Marchetti C, de Felice F, Palaia I, Perniola G, Musella A, Musio D et al (2014) Risk-reducing salpingo-oophorectomy: a meta-analysis on impact on ovarian cancer risk and all cause mortality in BRCA 1 and BRCA 2 mutation carriers. BMC Womens Health 14:150

Marchetti C, de Felice F, Boccia S, Sassu C, Di Donato V, Perniola $G$ et al (2018) Hormone replacement therapy after prophylactic risk-reducing salpingo-oophorectomy and breast cancer risk in BRCA1 and BRCA2 mutation carriers: a meta-analysis. Crit Rev OncolHematol 132:111-115

Mavaddat N, Antoniou AC, Mooij TM, Hooning MJ, HeemskerkGerritsen BA, Noguès C et al (2020) Risk-reducing salpingooophorectomy, natural menopause, and breast cancer risk: an international prospective cohort of BRCA1 and BRCA2 mutation carriers. Breast Cancer Res 22(1):8

Parker WH, Broder MS, Chang E, Feskanich D, Farquhar C, Liu Z et al (2009) Ovarian conservation at the time of hysterectomy and longterm health outcomes in the nurses' health study. ObstetGynecol 113(5):1027-1037

Perri T, Lifshitz D, Sadetzki S, Oberman B, Meirow D, Ben-Baruch $\mathrm{G}$ et al (2015) Fertility treatments and invasive epithelial ovarian cancer risk in Jewish Israeli BRCA1 or BRCA2 mutation carriers. FertilSteril 103(5):1305-1312

Razavi P, Pike MC, Horn-Ross PL, Templeman C, Bernstein L, Ursin G (2010) Long-term postmenopausal hormone therapy and endometrial cancer. CancerEpidemiol Biomarkers Prev 19(2):475-483

Rebbeck TR, Levin AM, Eisen A, Snyder C, Watson P, CannonAlbright L et al (1999) Breast cancer risk after bilateral prophylactic oophorectomy in BRCA1 mutation carriers. J Natl Cancer Inst 91(17): 1475-1479

Rebbeck TR, Friebel T, Wagner T, Lynch HT, Garber JE, Daly MB et al (2005) Effect of short-term hormone replacement therapy on breast cancer risk reduction after bilateral prophylactic oophorectomy in BRCA1 and BRCA2 mutation carriers: the PROSE Study Group. J ClinOncol 23(31):7804-7810

Rebbeck TR, Kauff ND, Domchek SM (2009) Meta-analysis of risk reduction estimates associated with risk-reducing salpingo-oophorectomy in BRCA1 or BRCA2 mutation carriers. J Natl Cancer Inst 101(2):80-87

Rocca WA, Grossardt BR, de Andrade M, Malkasian GD, Melton LJ (2006) Survival patterns after oophorectomy in premenopausal women: a population-based cohort study. Lancet Oncol 7(10):821-828

Rossouw JE, Prentice RL, Manson JE, Wu L, Barad D, Barnabei VM et al (2007) Postmenopausal hormone therapy and risk of cardiovascular disease by age and years since menopause. JAMA 297(13):1465-1477

S3-Leitlinie peri- und postmenopause-diagnostik und interventionen: AWMF-registernummer: 015-062; 2020.

Segev Y, Iqbal J, Lubinski J, Gronwald J, Lynch HT, Moller P et al (2013) The incidence of endometrial cancer in women with BRCA1 and BRCA2 mutations: an international prospective cohort study. GynecolOncol 130(1):127-131

Segev Y, Rosen B, Lubinski J, Gronwald J, Lynch HT, Moller P et al (2015) Risk factors for endometrial cancer among women with a BRCA1 or BRCA2 mutation: a case control study. Fam Cancer 14(3):383-391

Siyam T, Ross S, Campbell S, Eurich DT, Yuksel N (2017) The effect of hormone therapy on quality of life and breast cancer risk after risk-reducing salpingo-oophorectomy: a systematic review. BMC Womens Health 17(1):22

Network TAC, Centre NBC (2004) Clinical practice guidelines for the management of women with epithelial ovarian cancer. National Breast Cancer Centre, Camperdown, NSW

Vermeulen RFM, Korse CM, Kenter GG, Brood-van Zanten MMA, van Beurden M (2019) Safety of hormone replacement therapy following risk-reducing salpingo-oophorectomy: systematic review of literature and guidelines. Climacteric 22(4):352-360

Wagner U, Reuß A (2019) S3-Leitlinie "Diagnostik, Therapie und Nachsorge maligner Ovarialtumoren": Leitlinienprogramm Onkologie, Deutsche Krebsgesellschaft, Deutsche Krebshilfe, AWMF: Langversion 3.0, AWMF-Registernummer: 032/035OL.

Publisher's Note Springer Nature remains neutral with regard to jurisdictional claims in published maps and institutional affiliations. 